\title{
THE MODELLING AND CONTROL OF THE DRIVE SYSTEM OF AN ACKERMANN ROBOT USING GA OPTIMIZATION
}

\author{
E. I. Adamu1, ${ }^{*}$, M. O. Afolayan ${ }^{2}$, S. Umaru ${ }^{3}$ and D. K. Garba ${ }^{4}$

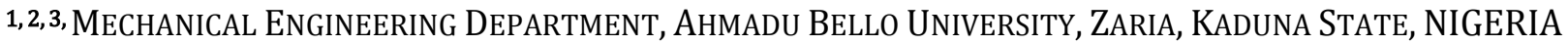

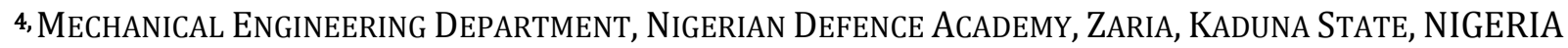 \\ E-mail addresses: ${ }^{1}$ ezekiel.adamuiyako@yahoo.com, ${ }^{2}$ tunde_afolayan@yahoo.com, ${ }^{3}$ bnumar1@gmail.com, \\ 4 dkgaruba2@gmail.com
}

\begin{abstract}
This paper provides the mathematical modelling and control optimization, of the drive system of an Ackermann four wheeled autonomous robot, with Genetic algorithm used for tuning the proportional, integral and derivative (PID) Controller parameters. The aim and main objective of this work is focused on the control of the driving speed input from the rear wheels of the robot and control. The robot drive in proportion to obstacle input ahead of the four wheeled chassis using genetic algorithms. A controlled platform that can be deployed for driverless vehicle in the nearest future and military unmanned vehicle is our major concern. The controlled system response stabilized in 0.675 seconds, after exciting the system with a step response. Variation for the system also shows, that the cost function was minimized or adjusted to obtain optimal PID parameters as Proportional $(P)=12.671$, Integral $(I)=$ 0.399 , Derivative $(D)=1477561$, at a value of $9.6778 * 10^{4}$.
\end{abstract}

Keywords: Ackermann steering, modelling, optimization, Genetic Algorithm, control, PID, driverless vehicle.

\section{INTRODUCTION}

Robotics is the science and technology of robots, which includes their design, manufacture, and application. Robotic researches are either abstract or biomimetic (biologically inspired) [1]. A robot is a mechanical agent which senses and manipulate its environment, moves around and can exhibit intelligent behaviours [2]. Therefore, the utilization of robots in areas of high risk jobs even in developing nations, is highly recommended. It was argued that improved health, safety and environmental performance is brought about by robots $[3,4]$ in their design using wheel control chassis which made use of differential method of wheel control for their motion.

Proportionally controlled steering mechanism is an automated mobile conveyor designed to transport materials. Most of them require guided path, although current developments are eliminating guide paths and are almost achieving a complete autonomous control [5]. Autonomous motion planning without collision with objects around the industry, farm or hospital at cheap cost is a challenge [6]. Inertia in Ackermann robot, poses a barrier to proper control however, during chassis motion unavoidable trajectory deviations between current position and requested trajectory occurs. That is because of path tracking control imperfection using wheels velocity and fault variables from environment such as terrain roughness, friction forces changes between wheels and terrain and so forth. A rocker type coal mine rescue robot [7] consisting of 4 wheels drive with bevel gear mechanism was proposed by [7]. The rocker suspension provides the stable mechanism for drive in all type of terrains. Similarly, in 2012, rocker-bogie mechanism was used in robot, which enhanced robots locomotion, abilities and flexibility. Then the Taguchi method was employed as an optimization tool to make the trajectory of Centre of Mass (COM) close to the straight line while all wheels are in contact with the ground during climbing stairs [8]. Autonomous vehicle are necessary in the military, experimental unmanned vehicle for terrain characterization which weighed about 3,400 pounds, and had full-time four-wheel drive had been deployed into rugged terrain for performance investigations [9]. The robot used in this work (Redface) is a four wheel Ackermann robot and is driven by a rear wheel engine. Such robots exhibit all the driving challenges found in rear wheel drive vehicles $[11,12]$. This design have serious impact on the steering and the control of the entire system. 


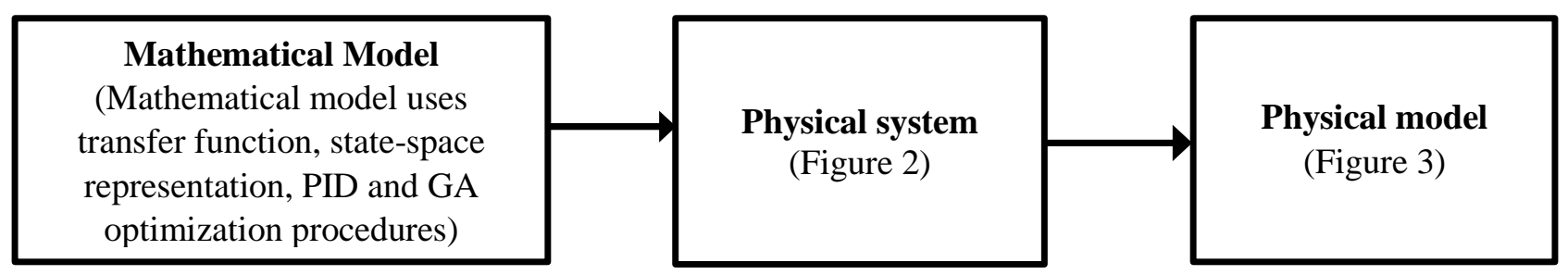

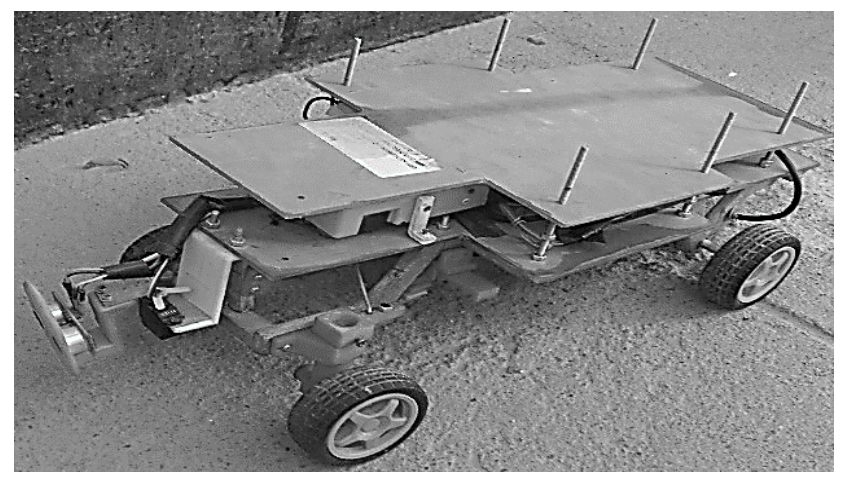

Figure 1 The developed robot (Red face)[7]

The aim of this work is to develop a mathematical model of the rear wheel drive and to optimize its control system using genetic algorithm.

This work is justified because, the input speed and braking control from the rear wheel of an Ackermann robot is very paramount in the directional control movement of these robots; since the drive speed determines the input angle of rotation for accurate steering of unmanned robots. Therefore it is highly essential to carry out a mathematical modelling for this robot.

\section{MODELLING PROCEDURE}

The robotic platform used for this research is a four wheel vehicle, figure1, code named Red face developed at the Ahmadu Bello University Zaria by Adamu Ezekiel [7].it was developed for this work. Figure 2 is the SolidWorks ${ }^{\circledR}$ CAD model of the Red face. The plastic parts labelled with PP are 3D printed. The Ackermann steering is also indicated in figure 2 . The control input into the robot is the linear velocity $U_{1}$ of the rear wheels, this input has serious impact on the steering and the control of the entire system. The modelling procedure followed in this work is outlined in figure 3; the physical system, is shown in figure 1, the physical model is shown in figure in figure 3 , and the mathematical model uses transfer function, state-space, PID and GA optimization procedures. All figures used on this work are dimensionless unless it is stated.

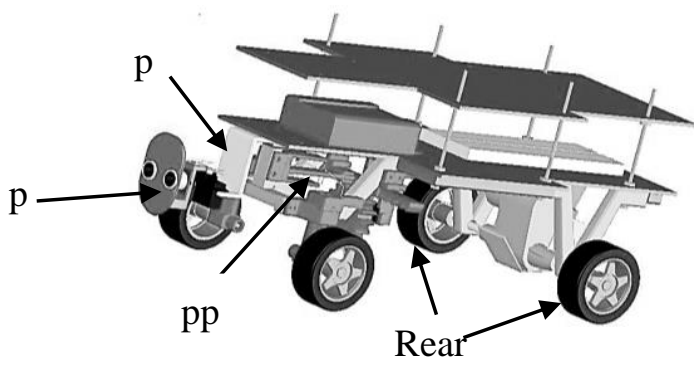

Wheels

Figure 2 CAD Model of Redface Robot[7]

\subsection{Creating the Mathematical Model of the robot rear wheel}

The mathematical model for Red face begins with the 370 DC motor which is the motive force for driving the Robot. The Robot body mass is approximated to be a load to be driven by the engine, although the rear axle comprises of a differential gear system with a gear ratio of $1: 1$

\subsection{Developing the transfer function and State Space Model for the Rear Wheel Motor}

The driving system of Redface is actuated by a DC motor, and Figure 3.0, shows the schematics of the motor system, with the electrical input being transformed into mechanical output on the shaft. Subsequently, it follows that equations are obtained from this same schematic relationship where, the motor torque, $\boldsymbol{T}$, is related to the armature current, $\boldsymbol{i}$, by a constant factor $\boldsymbol{K}_{\boldsymbol{t}}$, The back EMF, $\boldsymbol{e}$, is linked to the rotational velocity by the following equations: First the electrical voltage in the electrical part is transformed by the motor into the mechanical parts with the assumption of no losses. Using Kirchhoff's law on the diagram. Nomenclature and there descriptions are given on Table 1.

The voltage and the backward EMF of the DC motor is given by;

$$
V=L \frac{d i}{d t}+R i+e \quad \text { and } \quad\left(e=k_{e} \dot{\theta}\right)
$$

where $; k_{e}=$ electrical constant, $\dot{\theta}=$ motor angular speed,this implies 


$$
V=L \frac{d i}{d t}+R i+k_{e} \dot{\theta}
$$

when the inductance $\mathrm{L}$ is negligible or the armature current is constant, the rate of change of the current becomes zero and (2):

$$
V=R i+k_{e} \dot{\theta}
$$

The transfer function of this equation can be taken to be;

$$
V=(L s+R) I(s)+K s \theta(s)
$$

This electrical component causes the servo motor to produce a mechanical torque equivalent $T=K_{t} . i$ and $T=J \ddot{\theta}+b \dot{\theta}$ we equate the two torque we;

$$
J \ddot{\theta}+b \dot{\theta}=K_{t} \cdot i
$$

where; $\ddot{\theta}=$ is the angular acceleration

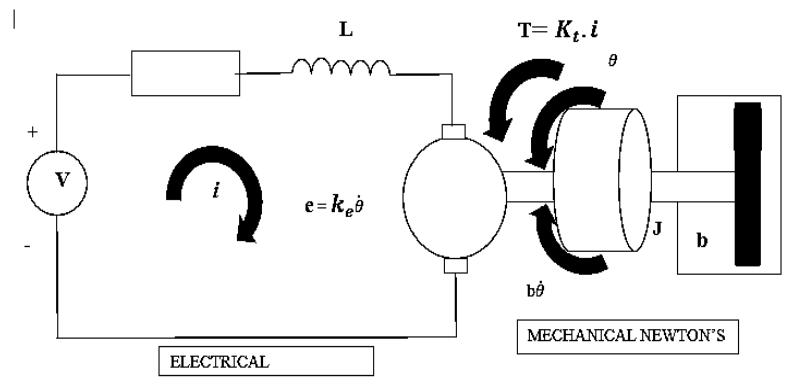

Figure 3 physical model of the robot rear wheel drive[7]

Table 1: nomenclature table

\begin{tabular}{cl}
\hline Symbol & Descriptions \\
\hline$T$ & Motor torque \\
$i$ & Armature current \\
$K_{t}$ & Motot torque constant \\
$e$ & Electromotive force (EMF) \\
$\mathrm{L}$ & Inductance \\
$\ddot{\theta}$ & Angular acceleration \\
$\dot{\theta}$ & Angular velocity \\
$\mathrm{V}$ & Input \\
$J_{L}$ & Moment of inertia of load \\
$J_{M}$ & Moment of inertia of motor \\
\hline
\end{tabular}

It is important to observe that mathematically, the mechanical component of anelectric motor at constant $\dot{\theta}$ will produce a zero angular acceleration. At such condition equation (5) will be reduced to the torque $T$ which is equivalent to;

$$
b \dot{\theta}=K_{t} . i
$$

Thus, the transfer function of equation (5) becomes;

$$
s(J s+b) \theta(s)=K I(S)
$$

$K_{t}$ (armature constant) is equal to $k_{e}$ (motor constant). From the Figure 2 we can write the following equations based on Newton's law combined with Kirchhoff's law, by eliminating $I(S)$ we can obtain the following transfer function, whereby the rotating speed is the output and the voltage is an input, The position is obtain by integrating $\dot{\theta}$.

$$
\frac{s \theta}{V}=\frac{K}{(J s+b)(L s+R)+K^{2}}
$$

Therefore dividing the Transfer function by s, in (8), we get:

$$
\frac{\theta}{V}=\frac{K}{s\left((J s+b)(L s+R)+K^{2}\right)}
$$

Where; moment of inertia of the rotor (J), damping ratio of the mechanical system (b), electromotive force constant $\left(\mathrm{K}=\mathrm{K}_{\mathrm{e}}=\mathrm{K}_{\mathrm{t}}\right)$, electric resistance $(\mathrm{R})$, electric inductance (L), input (V) i.e. Source Voltage, output $\theta$ (theta) i.e. position of shaft finally, the rotor and shaft are assumed to be rigid. This transfer function is used to obtain the dynamic equations in state-space form below:

$$
\begin{gathered}
\frac{d}{d t}\left[\begin{array}{c}
\theta \\
\dot{\theta} \\
i
\end{array}\right]=\left[\begin{array}{ccc}
0 & 1 & 0 \\
0 & -\frac{b}{J} & \frac{K}{J} \\
0 & -\frac{K}{L} & -\frac{R}{L}
\end{array}\right]\left[\begin{array}{c}
\theta \\
\dot{\theta} \\
i
\end{array}\right]+\left[\begin{array}{c}
0 \\
0 \\
1 \\
\frac{1}{L}
\end{array}\right] V \\
Y=\left[\begin{array}{lll}
1 & 0 & 0
\end{array}\right]\left[\begin{array}{c}
\theta \\
\dot{\theta} \\
i
\end{array}\right]
\end{gathered}
$$

Where;

$$
\begin{gathered}
\dot{X}=\frac{d}{d t}\left[\begin{array}{c}
\theta \\
\dot{\theta} \\
i
\end{array}\right], \quad A=\left[\begin{array}{ccc}
0 & 1 & 0 \\
0 & -\frac{b}{J} & \frac{K}{J} \\
0 & -\frac{K}{L} & -\frac{R}{L}
\end{array}\right], \\
X=\left[\begin{array}{c}
\theta \\
\dot{\theta} \\
i
\end{array}\right], B=\left[\begin{array}{c}
0 \\
0 \\
\frac{1}{L}
\end{array}\right], U=V, C \\
=\left[\begin{array}{ccc}
1 & 0 & 0
\end{array}\right], D=0
\end{gathered}
$$

When considering a DC motor under loading, the moment of inertia is a combine effect of the motors moment of inertia $J_{M}$ and the moment of inertia caused by the load $J_{L}$ therefore, the moment of inertia of a DC motor with load are[11];

$$
\begin{gathered}
J_{L}=\frac{1}{8} \mathrm{~m} D^{2} \\
J_{T}=J_{M}+J_{L}
\end{gathered}
$$

Based on the drive torque demanded, with moment of inertia $J_{T}$ as the new moment of inertia, the characteristic state space model of the drive system as given by [10], are revealed in (14) and (15). Thererfore, the Transfer Function of the rear motor is derived at (16).

$$
\begin{gathered}
\frac{d}{d t}\left[\begin{array}{c}
\theta \\
\dot{\theta} \\
i
\end{array}\right]=\left[\begin{array}{ccc}
0 & 1 & 0 \\
0 & -8.4 \times 10^{-3} & 2.54 \times 10^{-2} \\
0 & -1.43 \times 10^{2} & -1.02 \times 10^{7}
\end{array}\right]\left[\begin{array}{c}
\theta \\
\dot{\theta} \\
i
\end{array}\right] \\
+\left[\begin{array}{c}
0 \\
0 \\
8.7 \times 10^{6}
\end{array}\right] V \\
Y=\left[\begin{array}{lll}
1 & 0 & 0
\end{array}\right]\left[\begin{array}{c}
\theta \\
\dot{\theta} \\
i
\end{array}\right]
\end{gathered}
$$




$$
\mathrm{G}=\frac{220980}{S^{3}+1.02 e 07 S^{2}+8.568 e 04 s}
$$

transfer function of the rear wheel system

\subsection{System Model Verification}

The system in (xvii) is subject to a step input in order to verify the stability of the system. To compensate for unstable systems, controllers and algorithms or even both are used. The controller used in this work was a PID controller.

\subsubsection{PID Controller}

The rear drive system of the robot is approximated and connected to a PID controller is shown in figure 4, the $\mathrm{K}_{\mathrm{p}}$ is proportional gain constant, $\mathrm{K}_{\mathrm{i}}$ is Integral gain constant, $\mathrm{K}_{\mathrm{d}}$ is derivative gain constant. Our combined constants for the PID controller is given by (xvi).

$$
\mathrm{G}=\mathrm{K}_{\mathrm{p}}+\frac{\mathrm{K}_{\mathrm{i}}}{\mathrm{S}}+\mathrm{K}_{\mathrm{d}} \mathrm{S}
$$

Whereby $K_{p}$ is Proportional gain constant, $K_{i}$ is Integral gain constant, $K_{d}$ is Derivative gain constant. ZieglerNichols (ZN) tuning method was used.

The PID parameters arrived at by the ZN methods are set as the input initial parameters in the objective function of the GA to obtain the best possible parameters combination of the proportional, integral and the derivative controllers after 300 iterations subsequently.

\subsection{Genetic Algorithm Optimization of PID Parameters}

A sample population is taken with 50 initial population, these initial gains are crossed with a percentage of 0.8 after 300 iteration the results are being displayed with the best cost function value of the PID parameters. A terse collection of the GA parameters fed into the GA optimization tool box with the objective function (xix) are shown on Table 2 .

The objective function used in this work is estimated with an ITEA (Integral of time-weight absolute error), In this simulation the main objective is to minimize the objective function, which is the accumulated errors so as to select the best/optimum controller's parameters, the optimization criteria is the cost function, due to this purpose the cost function chosen is the ITAE Integral of time-weight absolute error which is based on Simpson's $1 / 3$ rule. The errors are the deviations of the Set point variables from the plants variable. Thus; it is necessary to note that the selection of the next generation is dependent on the cost function (fitness function).

$$
\begin{gathered}
\mathrm{ITAE}=\sum_{e(t)=r(t)-y(t)}(\mathrm{t} \cdot \operatorname{abs}(\mathrm{e}(\mathrm{t})) \mathrm{dt}) \\
e
\end{gathered}
$$

Where; $e(t)$ is error to be minimized, $r(t)$ is reference input to the plant, $y(t)$ is measured variable from the plant, $t$ is process time. The controller is multiplied with the error criteria in order to minimize the errors in the controller's gains as given by equation (20), which intuitively gives the approximated plant;

$$
\mathrm{W}=\left(\mathrm{kp}+\frac{\mathrm{ki}}{\mathrm{s}}+\mathrm{kd} . \mathrm{s}\right) \sum(\mathrm{t} \cdot \operatorname{abs}(\mathrm{e}(\mathrm{t})) \mathrm{dt}
$$

Based on the objective function, the optimization problem can be stated as Minimize objective Bounded by:

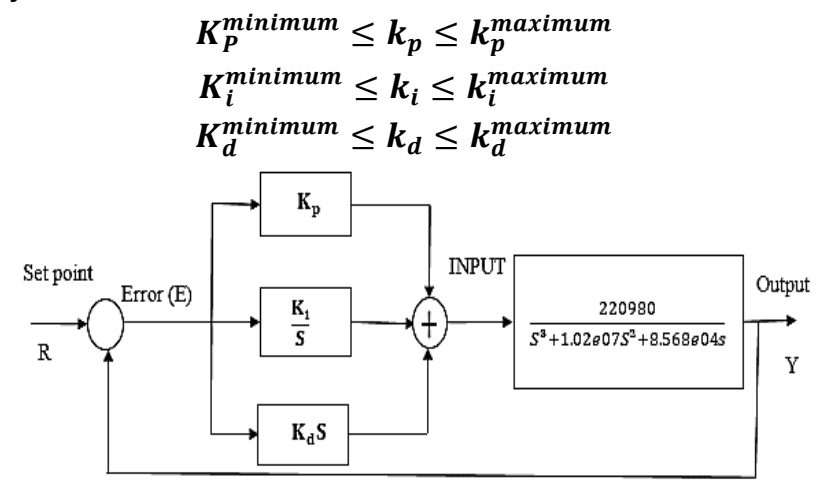

Figure 4: Tuning of the Response Using PID Controllers [7]

Table 2: GA parameters for optimization

\begin{tabular}{ll}
\hline GA Parameters & Values \\
\hline Population Size & 50 \\
Maximum iteration number & 300 \\
Mutation rate & $0.1 \%$ \\
Mutation Method & Uniform Mutation \\
Selection & 0.5 \\
Stall Generation & 50 \\
Cross over & 0.8 \\
Number of Cross over points & 3 \\
Fitness function & ITAE \\
\hline
\end{tabular}

The flow chart for the genetic algorithm on Figure 5, gives a summary of the selection processes in this work. The cost function is evaluated using the MATLAB function file in order to find the best cost function, it follows that the optimization terminates as seen in Figure 5.

\section{RESULT AND DISCUSSION}

Figure 6 is an unexpected behaviour of the reaction of our robot system to a step input as compared to ideal step input to systems, the excited model behaves as a linear system in the transient state with a significantly large amount of delay, over a very long time with an overshoot of 220000 at 45000 seconds. The poor response of our system to input depicts the realities that exist in real life applicable systems. The presence of noise affecting the model in reality, makes the 
system very unsafe for control application, hence, there is a serious need for compensation.

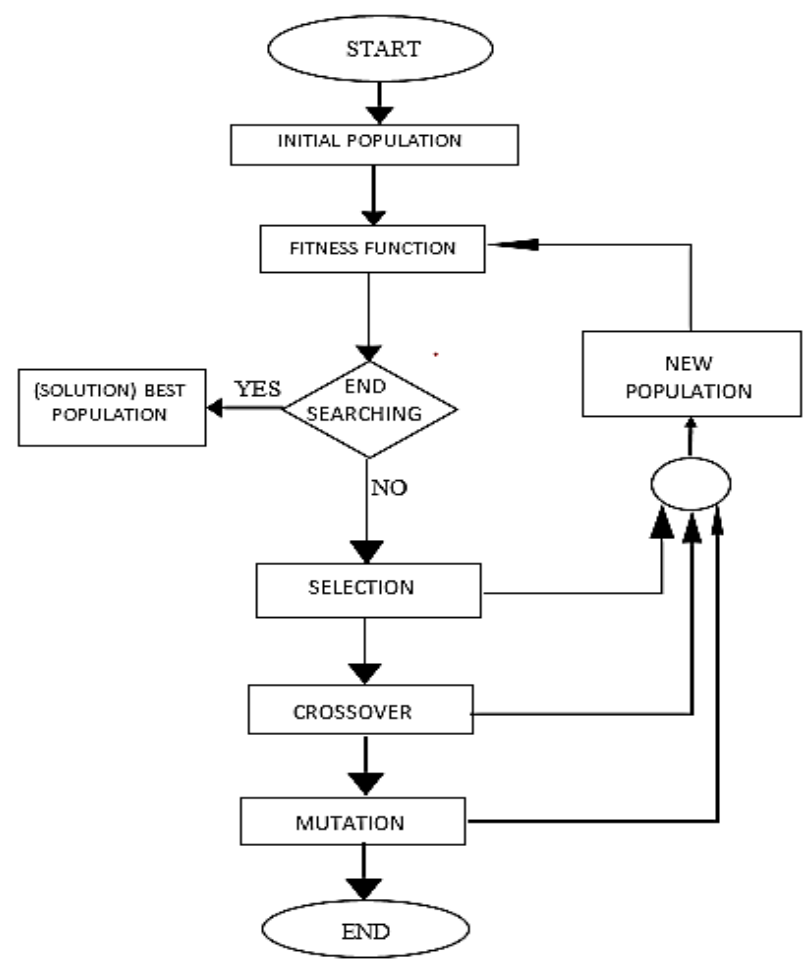

Figure 5.0: Genetic Algorithm Flow Chart

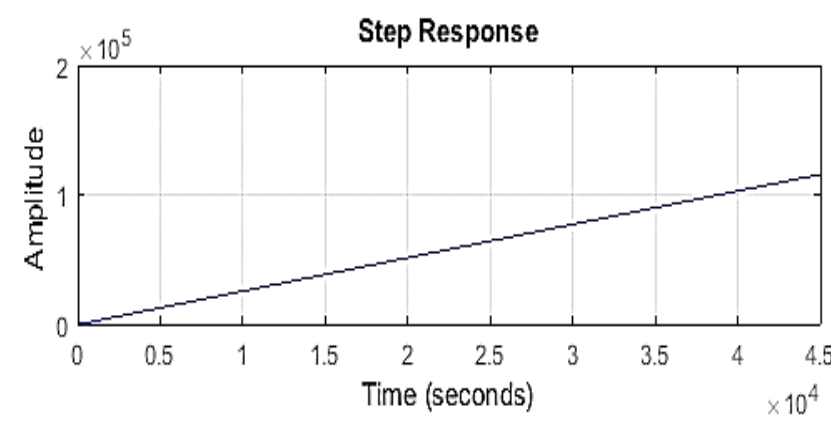

Figure 6: Step response of the uncontrolled system

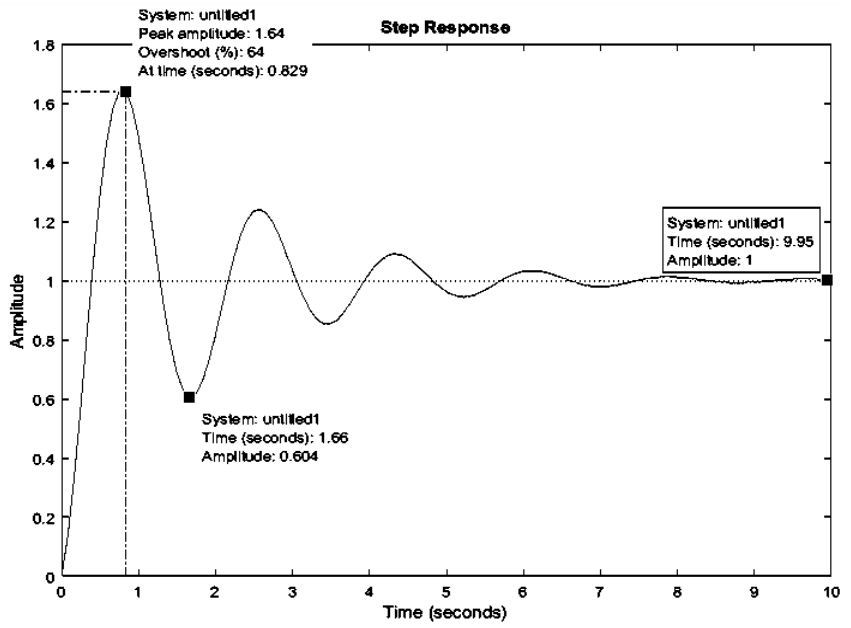

Figure 7: Using Zieglar-Nicholar's technique

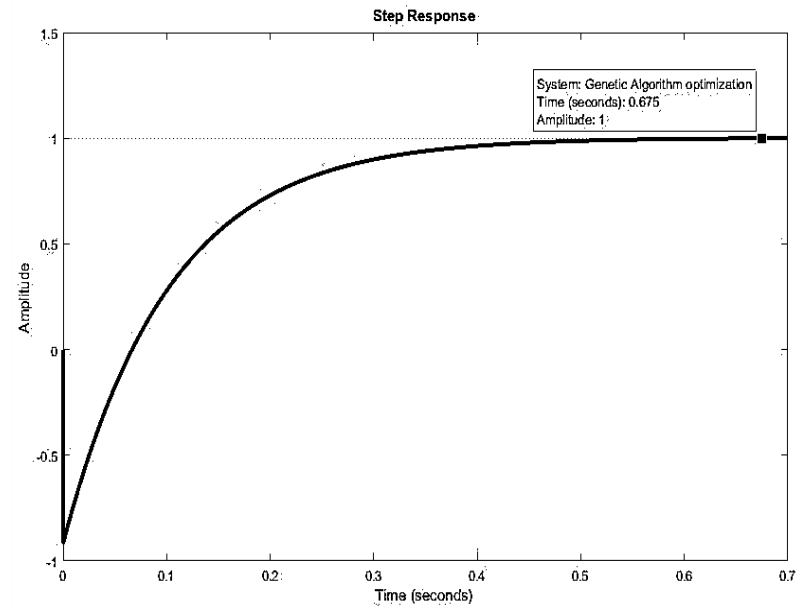

Figure 8: A variation of the step response of the rear input with time

The Ziegler-Nichol analytical tuning technique reveals the image on Figure 7.0, the system behaviour in the transient state shows the typical characteristics of several control systems in the transient state, with an overshoot of 1.64 at 0.829 seconds, the transient behaviour terminates at 9.95 seconds with the system attaining stability in regions beyond the transient state. Compensations with 9.95 seconds in this system are not appreciable enough; although they are far safer when compared with the previous system obtained in Figure 7.0 thus there is a need for further compensation

\subsection{Simulation for Tuning Robot with Genetic Algorithm} Variations are obtained as the test result of the software optimization for the rear input of our robot. The step response for the step controlled and the uncontrolled step response and then the cost function variation are also evaluated.

The step response variation of the rear motor for the reference and the controlled variations over time obtained via the use of MATLAB are shown on the figure 8.0. The uncontrolled response of the motor has unstable behaviours while the characteristics behaviour of the controlled response is stable at 0.675 seconds having a zero overshoot. Errors in the gains of the PID parameters is optimized by minimizing the errors with the best cost function. The PID controller gain parameters for the optimization where; Proportional $(\mathrm{P})=12.671$, Integral $(\mathrm{I})=-0.399$, Derivative $(D)=1477561$, the objective function value retrieved was $9.6778 * 10^{-4}$. The Figure9 reveals the fitness value of the cost function with each generation of offspring's, the disparity amongst the mean fitness and the best fitness values cancels out to merge at the thirtieth generation, the value for this point was 
0.000967781 and 0.000970716 , for both the best fitness and the mean fitness respectively.

The coherence continues to match all through the generations, this shows that significant fittest offspring are being produced from the thirtieth generation until the optimization process terminated. Figure $9.0 \mathrm{~B}$ is a bar chart of the new PID controller designed to compensate the system, the number of variation 1,2 and 3 are the proportional, integral and the derivative controllers respectively. Figure 9c this figure represents the individual offspring that emerged in all the generation, it is easy to note that hundreds of offspring combination evolved have been screened across all generation, in this simulation with the aim of selecting the most fittest candidate parameters combination for the best solution. Similarly, Figure 9d shows the selection function with the number of children by the 50 individuals showing the peak children within zero and 5 of the individuals having 15 number of children, this occurrence dies gradually, with sudden change between the $25^{\text {th }}$ and the $30^{\text {th }}$ individual and the $45^{\text {th }}$ and the $50^{\text {th }}$ individuals.

\section{CONCLUSION AND FUTURE WORK}

We designed a PID controller, and optimized the controller using genetic algorithm, by the optimization of the PID controllers, an efficient robot drive response was achieved in proportion to the inputs ahead of the robots path. This controller was able to minimize the reluctance of the robot to remain in rest when drive input is removed due to inertia, and such breaking system proffers a better control handling of the robot with higher precision. Closer distance gave faster response with the reversal effect on converse issues. In future, the robots plant can be approximated with a different controller to obtain a better response, other algorithms could also be tried for a better convergence; furthermore, the control implemented could be used for a real life model driverless vehicle, and for the control of military unmanned robots.

\section{ACKNOWLEDGEMENT}

This research work was supported by the Petroleum Technology Fund (PTDF) under grant 2016/2017 LSS scheme award no: PT/ED/LSS/MSc./AE1/08/16.

\section{REFERENCES}

[1] Afolayan, M. O. "Design and Development of a biologically inspired hyper-redundant robot joint Mechanism". PhD dissertation, Department of Mechanical Engineering. Ahmadu Bello University, Zaria Nigeria, pp. 38-49, 2014.

[2] Tom'as. "Artificial vision in Nao Humanoid robot". A Master's Thesis at Universitat Roviral I Virgili. Tracked Vehicle, U.S. Patent 7,493,976, pp 42, 46 and 52, February, 2009.

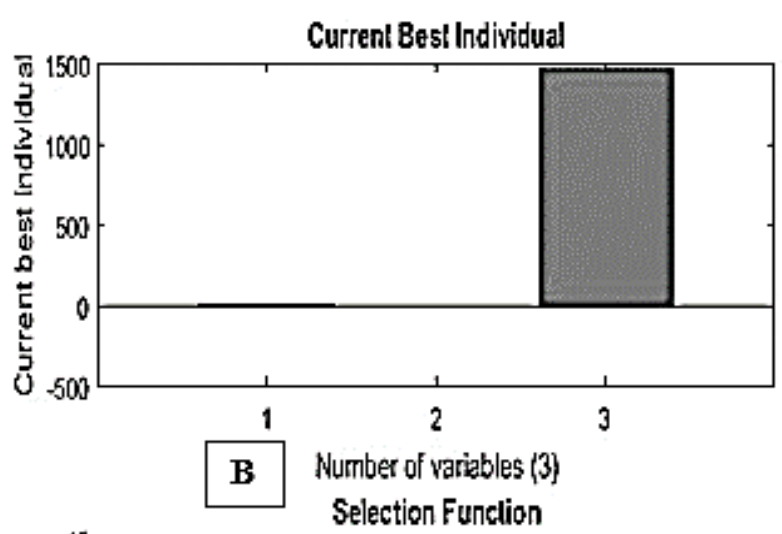

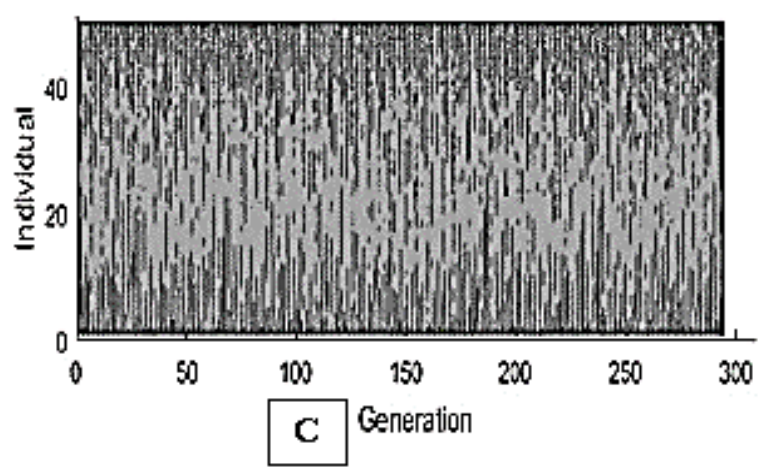

Best: 0.000967781 Mean: 0.000970716

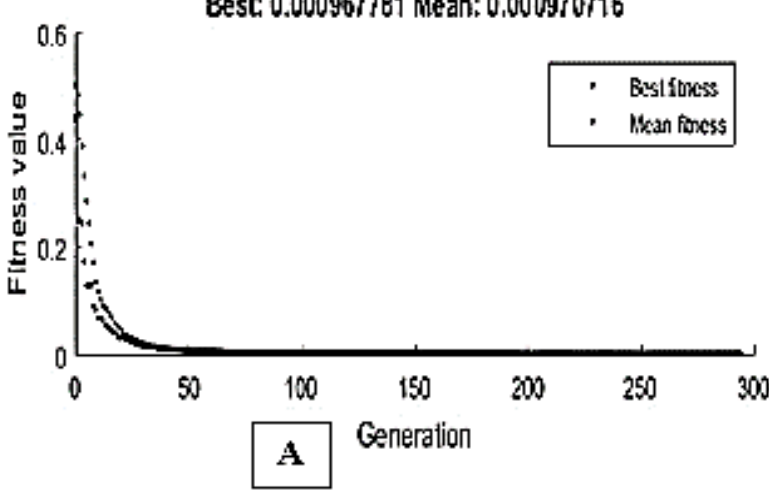

Figure 9: Variation of the GA's individual parameters and fitness values 
[3] Hashim, A. S., Tariq, D. M., \& Sinha, E. P. K. Application of robotics in oil and gas refineries. Int. J. Mech. Eng. Technol. (IJMET), 15, 01-08, 2014.

[4] Godoy, E. P., Tangerino, G. T., Tabile, R. A., Inamasu, R. Y., \& Porto, A. J. V. (2012). Networked control system for the guidance of a four-wheel steering agricultural robotic platform. Journal of Control Science and Engineering, 42012.

[5] Yunwang, L., Shirong, G., Hua, Z., Haifang, F., \& Jinke, G. A. O. Mobile platform of rocker-type coal mine rescue robot. Mining Science and Technology, 20 (3), 466-471, 2010.

[6] Spofford, J. R., and Gothard, B. M. Stopping distance analysis of ladar and stereo for unmanned ground vehicles. In Mobile Robots IX Vol. 2352, pp. 215-2271995.

[7] Adamu, Ezekiel Iyako, "Design, construction and testing of a proportionally controlled steering mechanism of robotic platforms for four wheel robotic application" MSc. Dissertation Department of Mechanical Engineering, Ahmadu Bello University Zaria. 2017.
[8] Leadshine (2015) "Motor torque calculation" retrieve at http://leadshineusa.com USA 6.42 AM 20/4/2017.

[9] Shneier. M and Bostelman .R (2015), Literature Review of Mobile Robots for Manufacturing. National Institute of Standards and Technology, U.S. Department of Commerce. Intelligent Systems Division Engineering Laboratory, 2015.

[10] Sharp R. S, Casanova D., and Symonds P., A Mathematical Model for Driver Steering Control, with Design, Tuning and Performance Results A Mathematical Model for Driver Steering Control, with Design, Tuning," Vehicle System Dynamics: International Journal of Vehicle Mechanics and Mobility, no. August 2010, pp. 37 41, 2010.

[11] Spofford, J. R., and B. M. Gothard. Stopping distance analysis of ladar and stereo for unmanned ground vehicles. Pp. 215-227 in Mobile Robots IX, Proceedings of SPIE Volume 2352. W.J. Wolfe and W.H. Chuneds. Bellingham, Wash: The International Society for Optical Engineering, 1994.

[12] Thomas Bräunl Embedded Robotics Mobile Robot Design and Applications with Embedded Systems, Springer-Verlag Berlin Heidelberg Germany, 2006. 